\title{
Low-power Inductive-coupling Transmitter Using Supply-insensitive Auxiliary Driving Switch under Supply-voltage Fluctuation
}

\author{
Yuya Nishio, Atsuki Kobayashi, and Kiichi Niitsu ${ }^{*}$ \\ Department of Electrical Engineering, Nagoya University, \\ IB-North 423, Furo-cho, Chikusa-ku, Nagoya 464-8603, Japan
}

(Received June 29, 2019; accepted April 22, 2020)

Keywords: biosensor, CMOS, healthcare IoT, inductive coupling, low-power technique, supply sensing, wireless transmitter

In this study, a constant-power inductive-coupling transmitter for low-power supply-sensing biosensing platforms is proposed. The proposed inductive-coupling transmitter is composed of two driving transistors with different inputs from different pulse generators. The two pulse generators have different working threshold voltages, and one of them works as the main driving switch, while the other works as an auxiliary driving switch, which turns on only at low supply voltages. This auxiliary driving technique realizes the constant power characteristics of the inductive-coupling transmitter because the auxiliary driving transistor only functions at low supply voltages, which reduces waste power at high supply voltages. To verify the effectiveness of the proposed inductive-coupling transmitter, a test chip is fabricated using $65 \mathrm{~nm}$ silicon-onthin-box (SOTB) CMOS technology. The test chip successfully demonstrates constant power characteristics, whereas the power consumed by the conventional inductive-coupling transmitter increases with an increase in the supply voltage.

\section{Introduction}

Healthcare Internet of Things (IoT) ${ }^{(1-3)}$ has been intensively investigated because of the increase in its demand by the ageing society. A stable power source is important to realize healthcare IoT. As candidate power sources of healthcare IoT, batteries, ${ }^{(4)}$ wireless power delivery, ${ }^{(5)}$ and energy harvesting ${ }^{(6)}$ systems have been reported. However, all of them have problems that should be resolved before they can be adopted as power sources. First, although batteries can provide stable power, they are not suitable from the viewpoint of safety. Moreover, they must be encased to implant them inside the human body and encased batteries are costly. In addition, there exist psychological problems concerning the implantation of batteries inside the human body. A wireless power delivery system is also an undesirable power source because a large antenna is required to receive large power. Moreover, it requires power management units such as AC-DC and DC-DC converters. Since it is difficult to obtain large stable power by energy harvesting, an energy harvesting system is also an unsuitable power source for

*Corresponding author: e-mail: niitsu@nuee.nagoya-u.ac.jp https://doi.org/10.18494/SAM.2020.2497 
healthcare IoT. On the other hand, biofuel cells $\mathrm{s}^{(7,8)}$ are considered promising power sources because they are safe and provide stable power. The structure and mechanism of biofuel cells are mentioned in previously reported works. ${ }^{(7,8)}$ It is reported that biofuel cells can generate an open-circuit voltage of $550 \mathrm{mV}$ with small variation. ${ }^{(7,8)}$ Moreover, they do not require an antenna, an $\mathrm{AC}-\mathrm{DC}$ converter, or a DC-DC converter. Therefore, the size and cost of the systems can be small.

The implementation of the supply-sensing $\operatorname{architecture}^{(9,10)}$ is a simple way to realize healthcare IoT, which is effective for sensing bioconcentration, to facilitate the monitoring of physical values for healthcare. In particular, the supply-sensing architecture is suitable for an energy-autonomous glucose monitoring system. Figures 1 and 2 show the block diagram and

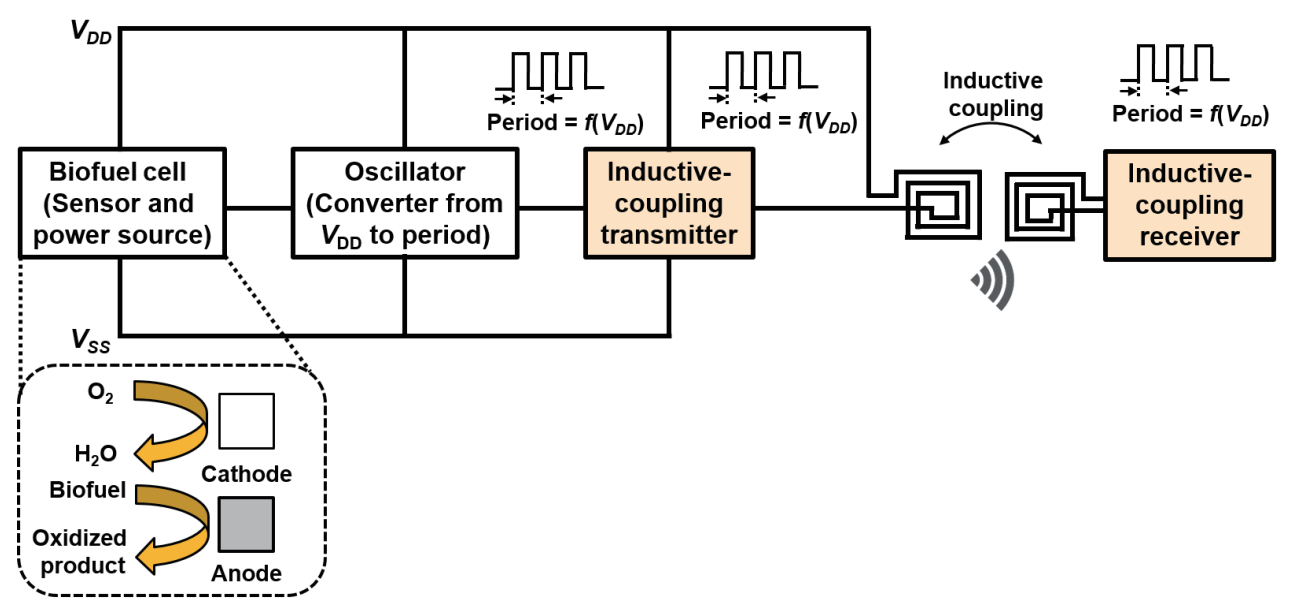

Fig. 1. (Color online) Block diagram of a supply-sensing architecture.
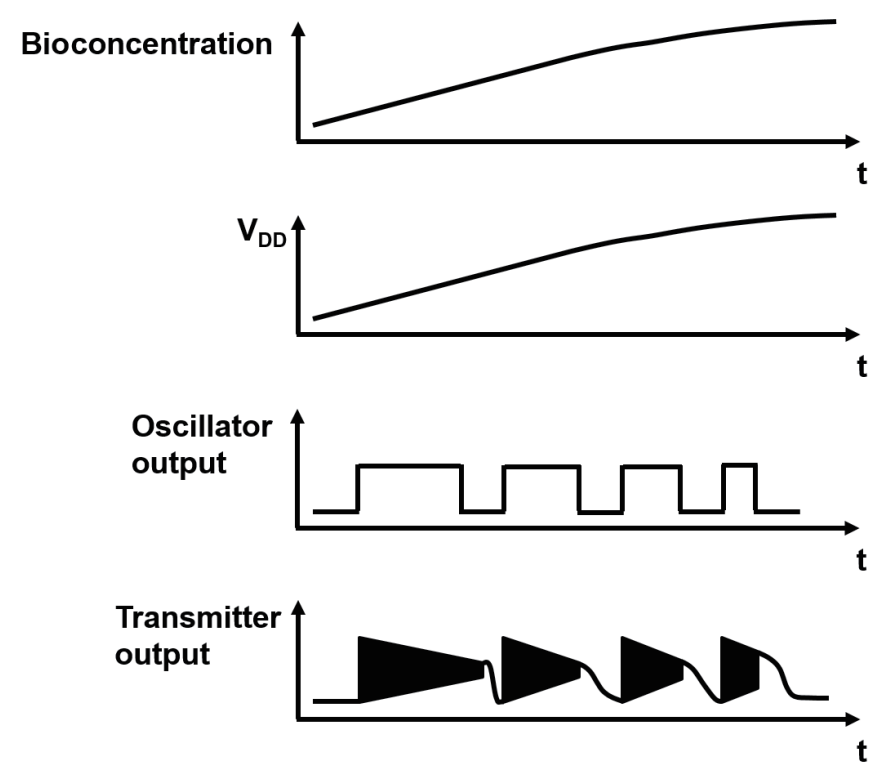

Fig. 2. Operational principle of a supply-sensing architecture. 
operational principle of a supply-sensing architecture, respectively. In the prototypes of the supply-sensing architecture, a biofuel cell is used as both a power source and a sensor. Thus, it is possible to convert bioconcentration to time-domain information (frequency) without requiring any power management circuits of analogue-to-digital converters (ADCs). Although we need an antenna for the transmitter, the antenna can be smaller than that for wireless power delivery. The supply-sensing architecture has been developed for cost reduction, but it is not optimized with regard to the power consumption. Since the supply voltage varies with the bioconcentration of the sensing target, the transmission power of the inductive-coupling transmitter varies. The transceiver is designed taking into consideration that the transmitter with the minimum supply voltage can achieve reliable communication. Thus, power transmitted at a high supply voltage results in overspecification (waste of power). In the case of implantable healthcare applications, small-footprint and low-power operations are the design requirements. Considering the supply-sensing architecture, the size of biofuel cells depends on the total power consumption of the system, and the power consumed by the transmitter predominantly accounts for a large portion of the total. Therefore, the development of an inductive-coupling transmitter with constant power transmission is in high demand for the realization of healthcare IoT.

In this paper, a new circuit design for achieving an inductive-coupling transmitter with constant transmission power is introduced. By introducing an auxiliary driving technique, which not only compensates for the degradation in transmission power at a low supply voltage but also cuts off waste consumption power at a high supply voltage, we developed a transmitter with constant transmission power. To verify the effectiveness of the proposed inductive-coupling transmitter, a test chip was designed and subsequently fabricated using $65 \mathrm{~nm}$ silicon-on-thin-box (SOTB) CMOS technology. The measurement results successfully demonstrate its constant power transmission capability.

In addition to its contribution to a conference publication, ${ }^{(11)}$ the present paper presents the effects of temperature and process variations on the ring oscillator and pulse generator. Moreover, the measurement results of the transmitters without and with an auxiliary driver are compared in this paper. On the basis of the results of this comparison, we discuss the advantages and disadvantages of the proposed auxiliary driving technique.

This paper is organized as follows: the proposed inductive-coupling transmitter using the auxiliary driving technique is introduced in Sect. 2. The chip design and measurement setup are summarized in Sect. 3. Section 4 presents the measurement results of the test chip. In Sect. 5, we discuss the advantages and disadvantages of the proposed technique. In Sect. 6, we conclude this paper.

\section{Proposed Inductive-coupling Transmitter Using Auxiliary Driving Technique}

\subsection{Concept and overall architecture}

Figures 1 and 2 show the block diagram and operational principle of a supply-sensing architecture with the inductive-coupling transmitter, respectively. In a supply-sensing architecture, biofuel cells can be used as both a power source and a sensor, which simplifies 
the sensing system. Biofuel cells generate power by the reduction and oxidization of the enzyme at the cathode and anode, respectively. A biofuel cell generates voltage in the range from 100 to $500 \mathrm{mV}^{(8)}$ In the supply-sensing architecture, the supply voltage depends on the bioconcentration. Then, an oscillator converts the supply voltage to the period of the output signal. Therefore, the frequency of the inductive-coupling transmitter is determined by the bioconcentration. The period data is then transferred from the transmitter to the receiver via an inductive-coupling link. Therefore, in the supply-sensing architecture, the output frequency from the inductive-coupling transmitter contains information on bioconcentration.

Figure 3 shows the conceptual diagram of the technique proposed in this study. In the conventional inductive-coupling transmitter, the transmitted power increases with the supply voltage. Therefore, the transmitted power becomes higher than required because transmitters are designed for a minimum supply voltage, leading to power wastage and additional power consumption at high supply voltages and reduced power efficiency. Furthermore, in a supplysensing architecture, information on bioconcentration is contained in the output frequency of the inductive-coupling transmitter, and the additional transmission power at high supply voltages results in power wastage. However, owing to the newly introduced auxiliary driving technique, the proposed transmitter consumes constant transmission power even at a high supply voltage, thus reducing power wastage. Since transmission power predominantly accounts for a large portion of the total power of the entire system, the proposed inductive-coupling transmitter is suitable for reducing the power of the entire system. The proposed technique is also beneficial for reducing the size of biofuel cells, which is determined by the total consumption power.

(a) Conventional inductive-coupling transmitter (Nonconstant transmission power)
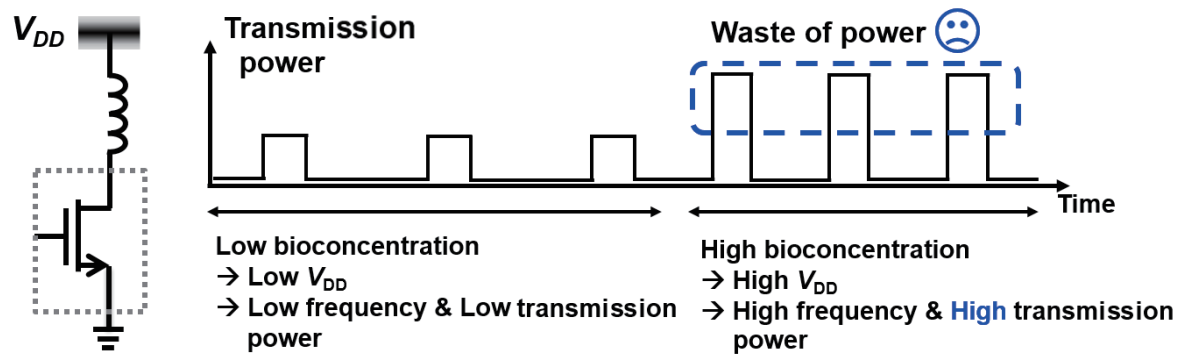

(b) Proposed inductive-coupling transmitter (Constant transmission power)

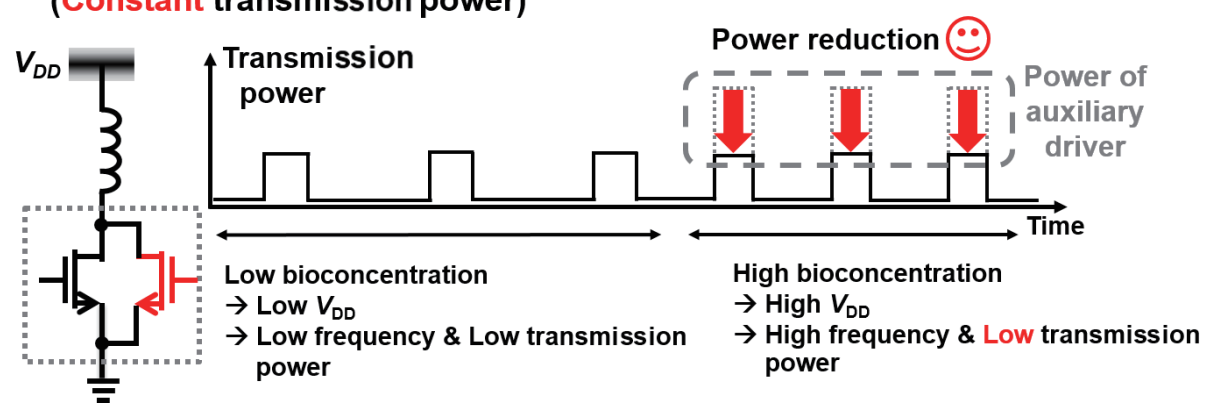

Fig. 3. (Color online) Conceptual diagram of proposed technique. 
Figures 4(a) and 4(b) show the circuit diagrams and corresponding power-voltage graphs of the conventional and proposed inductive-coupling transmitters, respectively. As shown in Fig. 4, the proposed inductive-coupling transmitter adopts an auxiliary driving transistor in addition to the main driving transistor, whereas the conventional inductive-coupling transmitter uses only one switch. The auxiliary driver (M2) supports the main driver (M1), which has advantages at both low and high input voltages. When the input voltage is low, the auxiliary driver assists transmission, facilitating transmission at lower input voltages than in the conventional inductive-coupling transmitter. When the input voltage is high, the auxiliary driver stops working, reducing power wastage and enabling lower power consumption than in conventional transmitters. The power consumption graph shows that the proposed inductive-coupling transmitter cuts off the waste power at a high supply voltage, thereby realizing constant power transmission.

\subsection{Circuit implementation}

Figure 5 shows the schematic of the proposed inductive-coupling transmitter with a ring oscillator. The proposed inductive-coupling transmitter differs from conventional transmitters in that it utilizes two driving transistors (main and auxiliary) in parallel and implements an additional control circuit. Although the required footprint of the proposed transmitter is larger than that of the conventional one that utilizes only one driver because of the additional control circuit, more advantages can be gained from this circuit. Furthermore, the size of the control circuit is much smaller than that of a driving transistor because it can be realized in the form of a digital circuit. Thus, the additional footprint is small enough to be negligible. Although

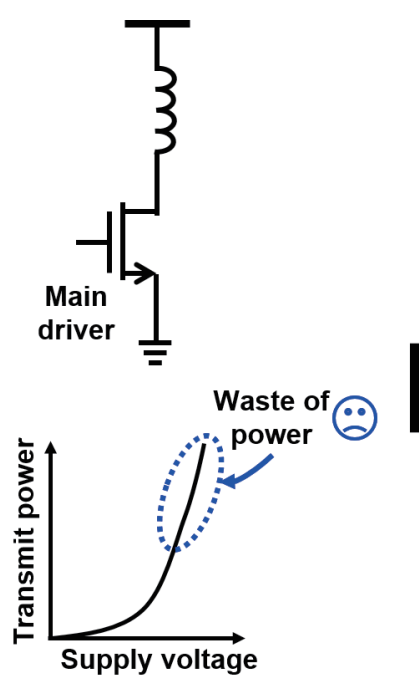

(a) Conventional transmitter (Nonconstant transmit power)

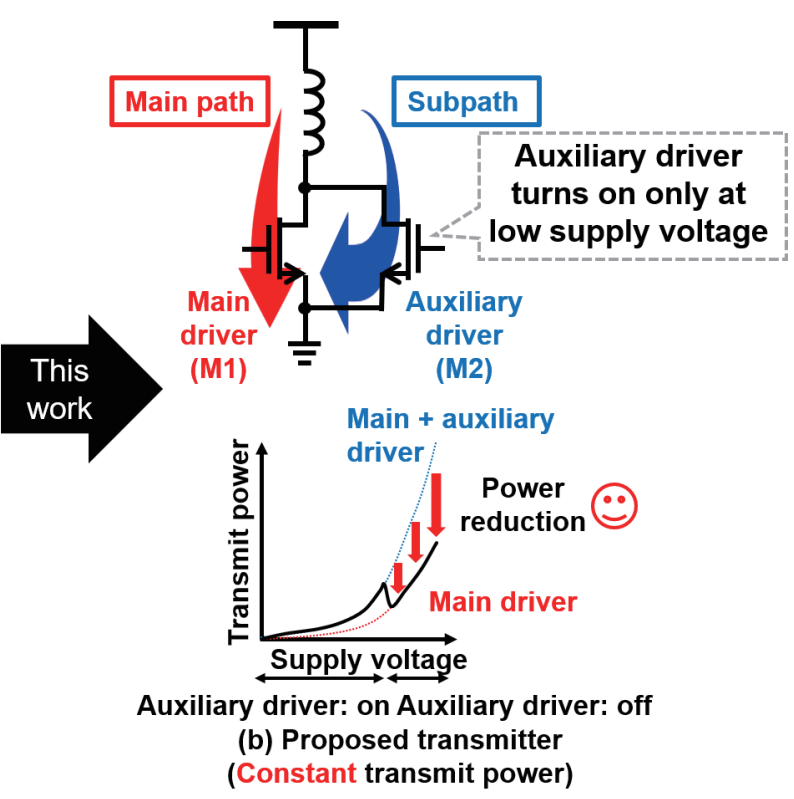

Fig. 4. (Color online) Circuit diagrams and corresponding power-voltage graphs of (a) conventional and (b) proposed inductive-coupling transmitters. 


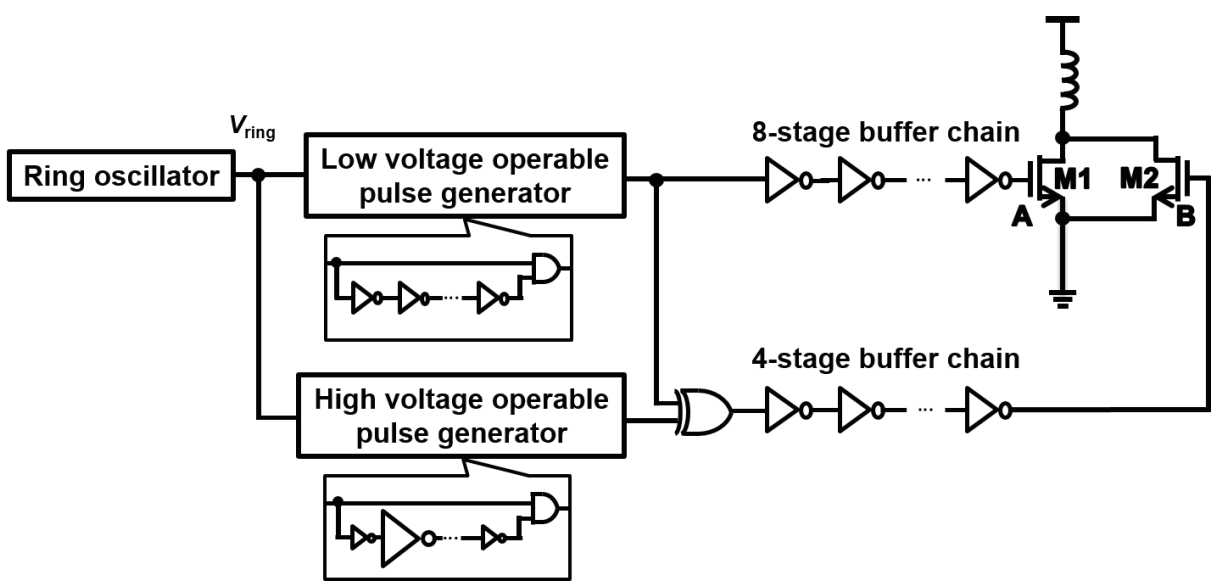

Fig. 5. (Color online) Schematic of proposed transmitter with ring oscillator.

output impedance varies with the switching of the auxiliary driving transistor, we consider that it is within an acceptable range and does not affect the circuit operation.

A ring oscillator, two pulse generators, buffer chains, an exclusive or (XOR) gate, two driving transistors, and an inductor compose the overall circuit. The two pulse generators are different in terms of the minimum operable supply voltage. The minimum operable supply voltage of one of the pulse generators is low, while that of the other is relatively high. The high-voltage operable pulse generator is realized by alternately connecting small and large inverters in series. Since driving a capacitor with a large capacitance requires a high supply voltage, the minimum operable supply voltage increases compared with that in the case of the pulse generator, in which similar inverters are connected. The minimum supply voltage $\left(V_{D D}\right)$ at which the pulse generator can work increases with the size ratio of the inverters. A large capacitance can also be realized with a varactor. However, we realized a large capacitance by increasing the inverter size in order to prevent an increase in circuit size. We also adopted a spiral inductor ${ }^{(12,13)}$ in the transmitter.

The number of inverter stages in pulse generators and buffer chains is also varied in order to change the delay time and therefore drive two transistors simultaneously for the realization of the auxiliary driving technique. The difference in the number of inverter stages also compensates the delay of the XOR gate. Although the delay time varies with the supply voltage, it does not affect the operation of the proposed inductive-coupling transmitter because biofuel cells generate power that is stable enough to suppress variations within the tolerance range. ${ }^{(7,8)}$

The size of the main and auxiliary driving transistors can be determined by considering the characteristics of consumption power and supply voltage. If the size of the main driving transistor is small, the consumption power at all supply voltages decreases. On the other hand, if the size of the auxiliary driving transistor is small, less flat consumption power characteristics against supply voltage are obtained. By increasing the relative size of the auxiliary driving transistor against the main driving transistor, we can obtain flatter characteristics.

Although we connected only one driving transistor as an auxiliary driver in this work, higher constant-power characteristics can be achieved by connecting more transistors in 
parallel. Therefore, the proposed architecture is scalable. However, as the number of auxiliary transistors increases, the circuit area also increases. Therefore, the number of auxiliary driving transistors can be determined by considering the trade-off between the performance and the area overhead.

\subsection{Operational principle}

Figure 6 shows the operational diagram of the proposed inductive-coupling transmitter. The auxiliary driving technique is realized by connecting the auxiliary driving transistor, which works only at a low supply voltage, in parallel with a conventional driving transistor. The auxiliary driving architecture provides a main path and a subpath for a transmission current. In addition to the minimum operable supply voltage for the pulse generator, we adjust the number of inverter stages in pulse generators and buffer chains to turn on two driving transistors simultaneously in order to maximize the power efficiency.

As shown in Fig. 6(a), when the supply voltage is low, the low-voltage operable pulse generator outputs a high voltage, while the high-voltage operable pulse generator outputs a low voltage. The outputs of the pulse generators are input to the XOR gate, making the auxiliary driving transistor compensate for the necessary driving power for transmission. Both the output voltages of the low- and high-voltage operable pulse generators are high at a high input voltage, in contrast to those at a low input voltage, as shown in Fig. 6(b). Thus, the auxiliary driving transistor M2 does not function at high input voltages because the XOR gate outputs a low voltage when the two input voltages to the XOR gate are both high.

(a) Low supply voltage

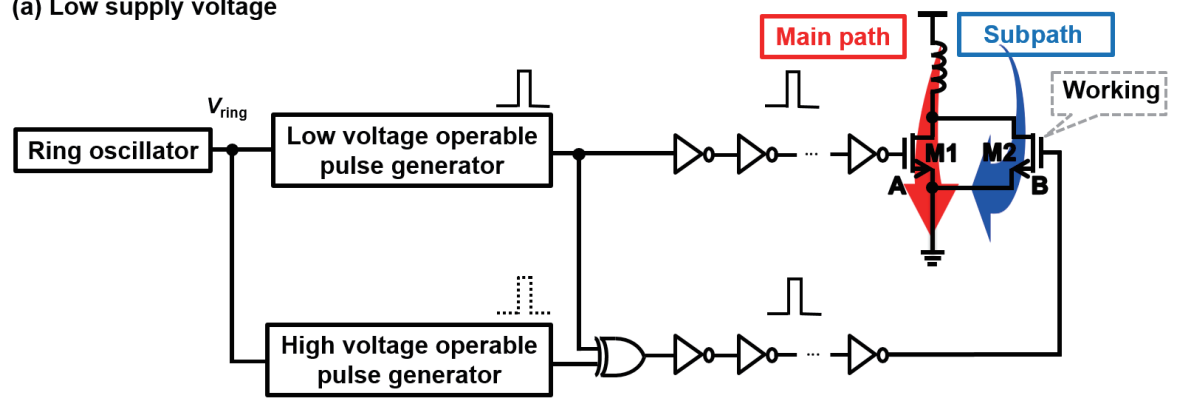

(b) High supply voltage

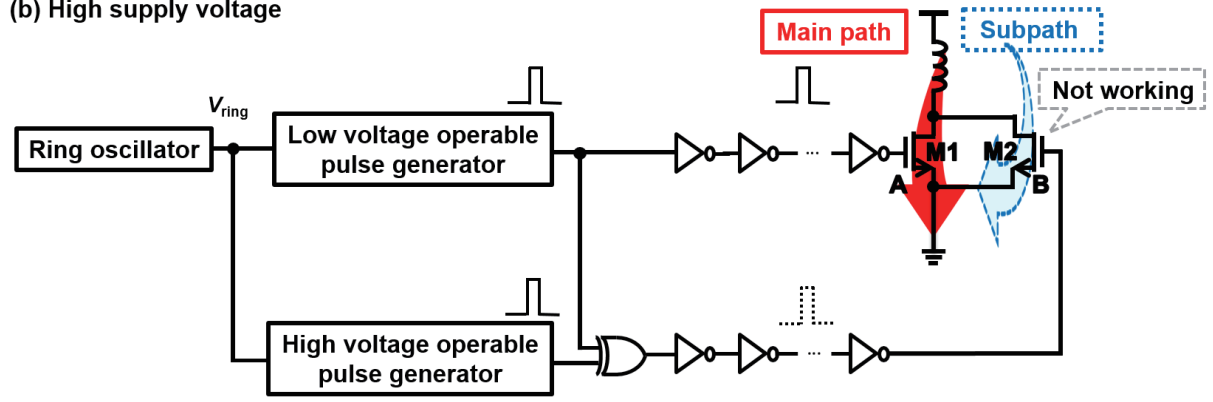

Fig. 6. (Color online) Conceptual operational circuits of proposed inductive-coupling transmitter at (a) low and (b) high input supply voltages. 
In the inductive-coupling transmitter, the power consumption of the driving transistor accounts for the majority of the total power consumption in the circuit. In the conventional inductive-coupling transmitter, the power of the circuit increases with the supply voltage. However, in the proposed transmitter, the power of the circuit does not simply increase with the supply voltage, because the auxiliary driving transistor does not function if the voltage is above a certain limit (the minimum operable voltage of the high-voltage operable pulse generator). By disabling the auxiliary driving transistor, we can limit power wastage, thereby reducing the total power consumption of the circuit and enhancing the power efficiency. Furthermore, owing to the auxiliary driving transistor, which compensates the necessary power to be transmitted at low input voltages, the proposed inductive-coupling transmitter transmits the power at a lower input voltage than the conventional inductive-coupling transmitter.

\subsection{SPICE simulation}

Figures 7 and 8 show the transient simulation waveforms of the voltages and powers of the main and auxiliary driving transistors of the proposed transmitter at supply voltages of 0.4 and $0.45 \mathrm{~V}$, respectively. These figures also show that the main and auxiliary driving transistors turn on simultaneously because of the adjustment of the number of inverter stages in pulse generators and buffer chains. Figures $7(\mathrm{a}), 7(\mathrm{~b}), 8(\mathrm{a})$, and $8(\mathrm{~b})$ show that the auxiliary driving
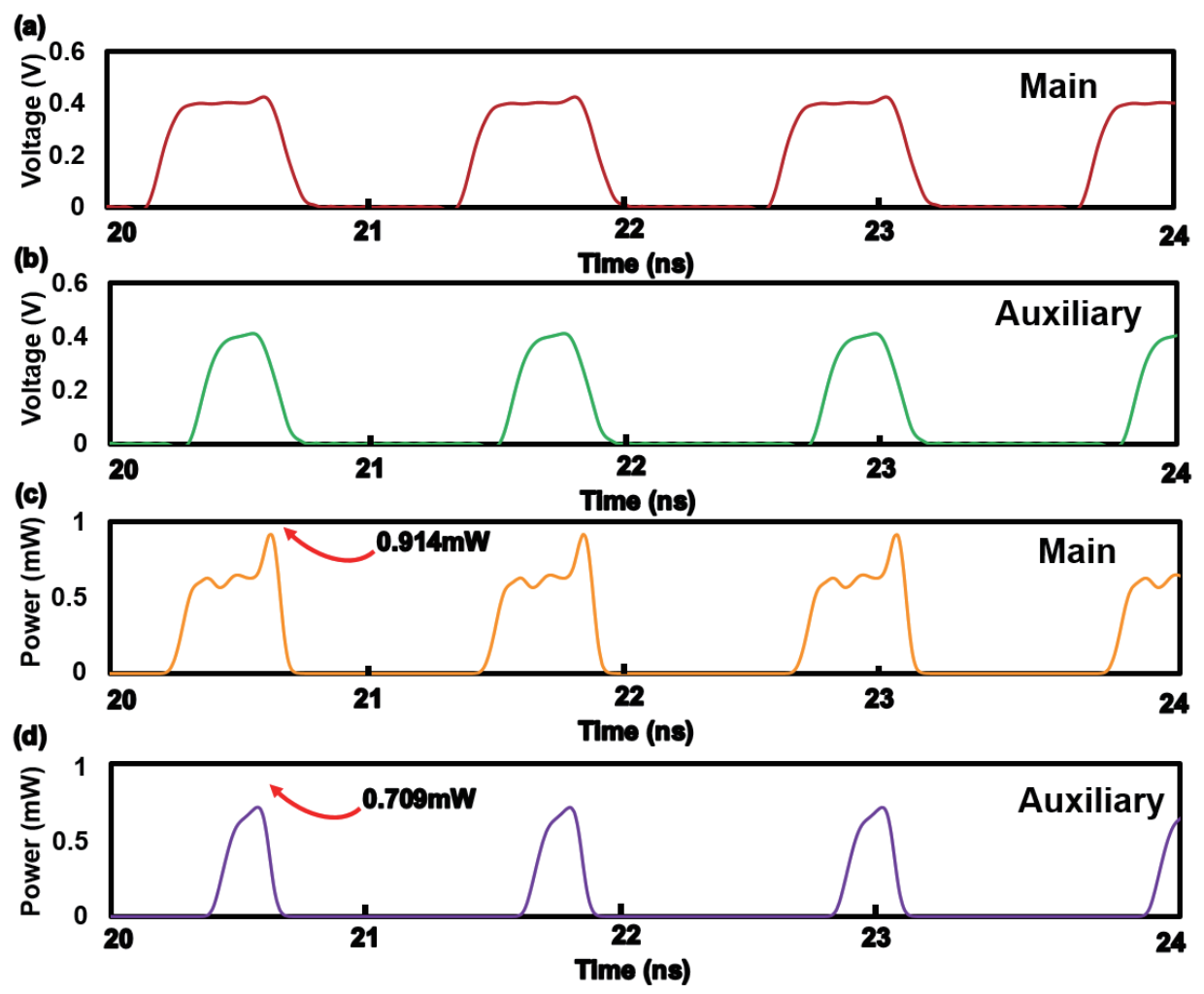

Fig. 7. (Color online) Transient simulation of gate voltage and power of main and auxiliary driving transistors at supply voltage of $0.4 \mathrm{~V}^{(11)}$ 
(a)

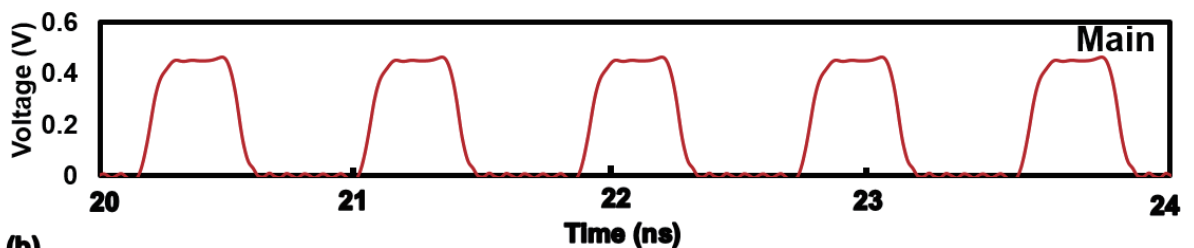

(b)

Time (ns)
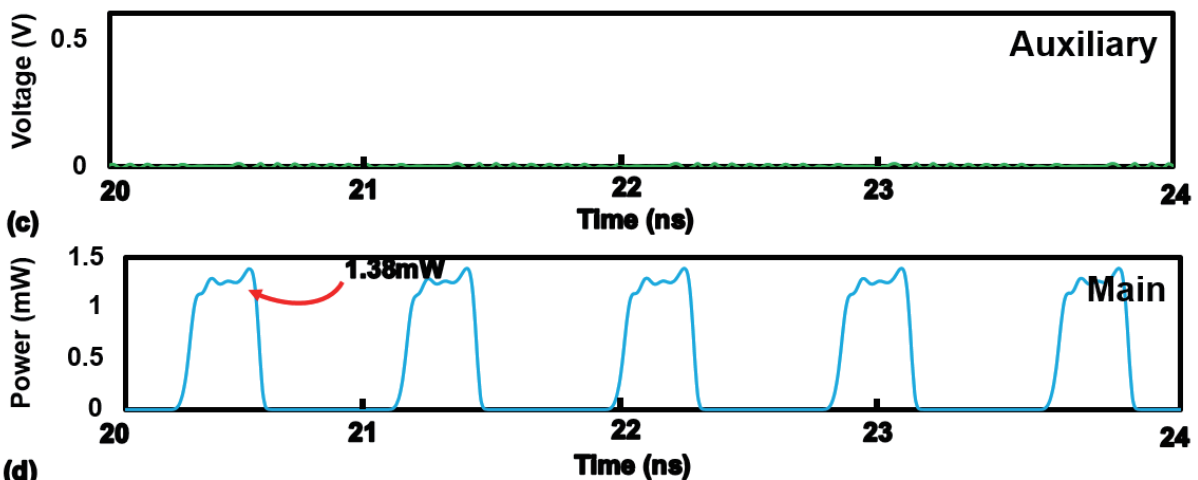

(d)

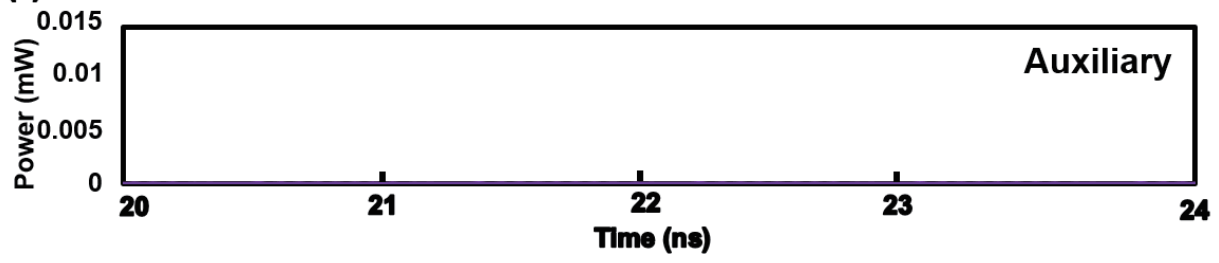

Fig. 8. (Color online) Transient simulation of gate voltage and power of main and auxiliary driving transistors at supply voltage of $0.45 \mathrm{~V}^{(11)}$

transistor M2 functions only when the supply voltage is $0.4 \mathrm{~V}$, whereas the main driving transistor M1 functions at both supply voltages of 0.4 and $0.45 \mathrm{~V}$. This is because of the two types of pulse generator (high-/low-voltage operable pulse generators) and the XOR gate. In other words, M2 functions only when the supply voltage is low to compensate for the reduced transmission power, as explained in Sect. 2.3. Since information on bioconcentration is included in the pulse interval of the output signal from the transmitter, the mismatch in the pulse width from the main path and subpath can be neglected, whereas the matching of the timing of the pulse from the main path and subpath is crucial. Hence, we adjusted the delay time using the buffer chain after pulse generators to ensure this. It is sufficient to consider only the powers of driving transistors because the power consumption of the control circuit is low and the majority of the power consumption of the proposed circuit is due to driving transistors.

Figures 7(d) and 8(d) show that the power of the auxiliary driving transistor is cut off because it does not function at high supply voltages. Owing to this effect, the total power consumption at high supply voltages can be reduced. In particular, the total power consumed by the main and auxiliary driving transistors is $1.62 \mathrm{~mW}$ for a supply voltage of $0.4 \mathrm{~V}$, whereas it is $1.38 \mathrm{~mW}$ for a supply voltage of $0.45 \mathrm{~V}$. On the other hand, the power of the conventional transmitter increases with the supply voltage. This implies that disabling the auxiliary driving transistor successfully reduces the waste power at high supply voltages. 
Figure 9 shows the supply voltage dependences of the frequency of the ring oscillator and pulse width of pulse generators. The black squares and line show the simulated frequency of the ring oscillator. Red and blue data show the pulse widths of the high- and the low-voltage operable pulse generators, respectively. The simulation was conducted at a temperature of $27{ }^{\circ} \mathrm{C}$. Although there is some degree of fluctuation, Fig. 9 shows that the frequency of the ring oscillator increases with the supply voltage. This means that the frequency of the ring oscillator can provide information on bioconcentration. Furthermore, the pulse width of pulse generators is stable at high supply voltages. Since biofuel cells generate stable voltages, variations in pulse width are negligible. Further improvement in the architecture of the ring oscillator, such as the addition of a phase-locked loop to suppress the effects of jitter and phase noise, is required.

Figure 10 shows the temperature dependence of the pulse width of pulse generators under process variations. The simulation was performed at a supply voltage of $0.3 \mathrm{~V}$. Red and blue data show the simulated results of the pulse widths of the low- and high-voltage operable pulse generators, respectively. We performed simulations at different corners to test the performance of the pulse generators under process variation. Figure 10 shows that the pulse width of the pulse generator varies with process variation. The calibration architecture used to suppress the variation will be reported in our future works. The pulse width also varies with the temperature. However, since the human body temperature is stable, the variations caused by the temperature are negligibly small.

\section{Test Chip and Measurement Setup}

\subsection{Test chip design}

To verify the effectiveness of the proposed inductive-coupling transmitter, a test chip was fabricated using $65 \mathrm{~nm}$ SOTB CMOS technology. We fabricated a circuit that is the same as

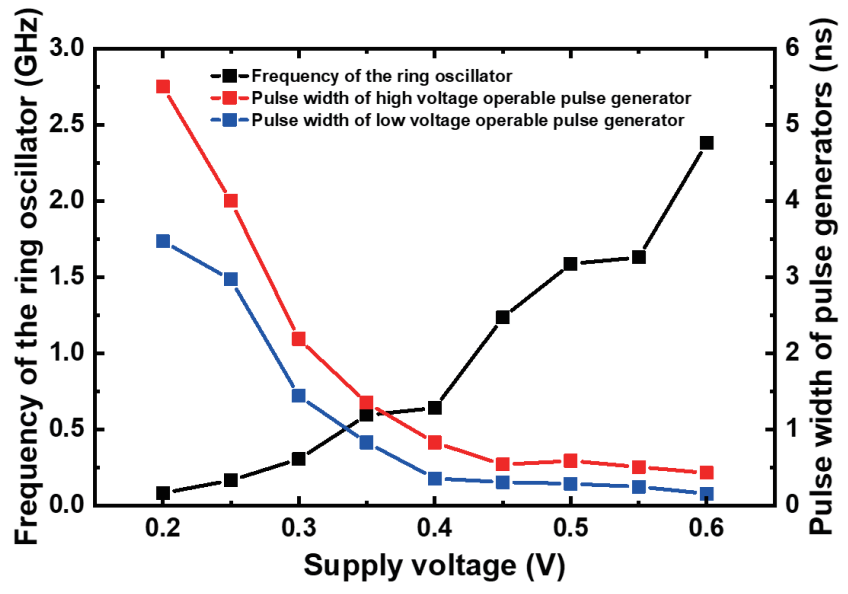

Fig. 9. (Color online) Simulated supply voltage dependences of frequency of ring oscillator and pulse width of pulse generators.

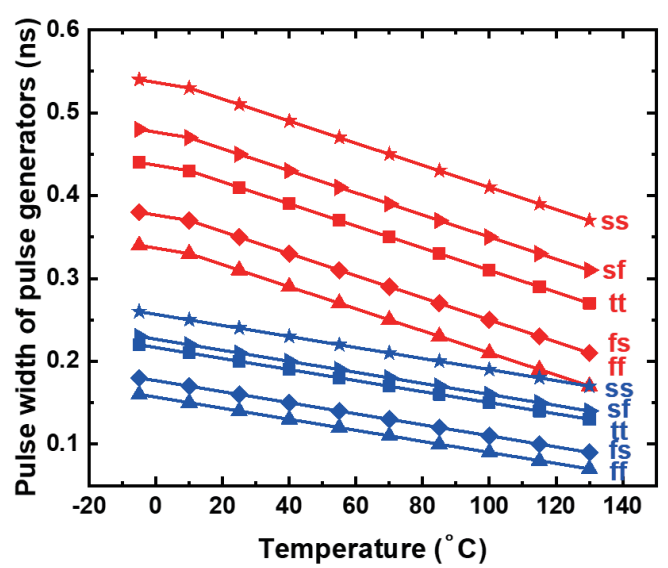

Fig. 10. (Color online) Simulated temperature dependence of pulse width of pulse generators under process variation. 
that shown in Fig. 5. Figure 11 shows the microphotograph and layout image of the test chip. The area occupied by the core circuit of the transmitter is $180 \mathrm{~mm}^{2}$, which is similar to that of conventional transmitters. Since the proposed technique requires additional pulse generators and logic circuits, the footprint is slightly larger than that of conventional techniques. However, the additional footprint is not greater than $10 \%$ of that of conventional techniques and is negligible.

\subsection{Measurement setup}

The measurement setup is shown in Fig. 12. An RF magnetic probe (Langer, near-field probe set XF1) was employed for detecting the transmission power. A source measure unit (Keysight, B2912A) and a sampling oscilloscope (Keysight, DSO8104A) were employed for feeding and capturing the signal, respectively. The RF magnetic probe was placed on top of the inductor and the communication distance was longer than $50 \mathrm{~mm}$.

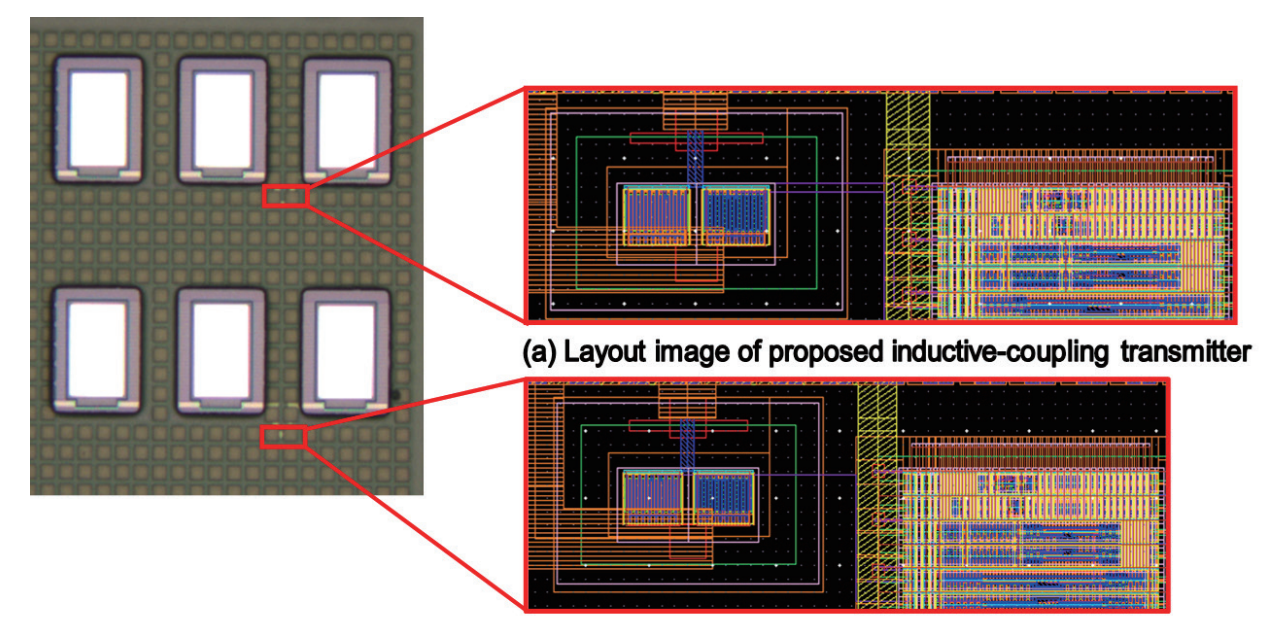

(b) Layout image of conventional inductive-coupling transmitter

Fig. 11. (Color online) Microphotograph and layout image of the prototype chip fabricated by $65 \mathrm{~nm}$ SOTB CMOS technology. ${ }^{(11)}$
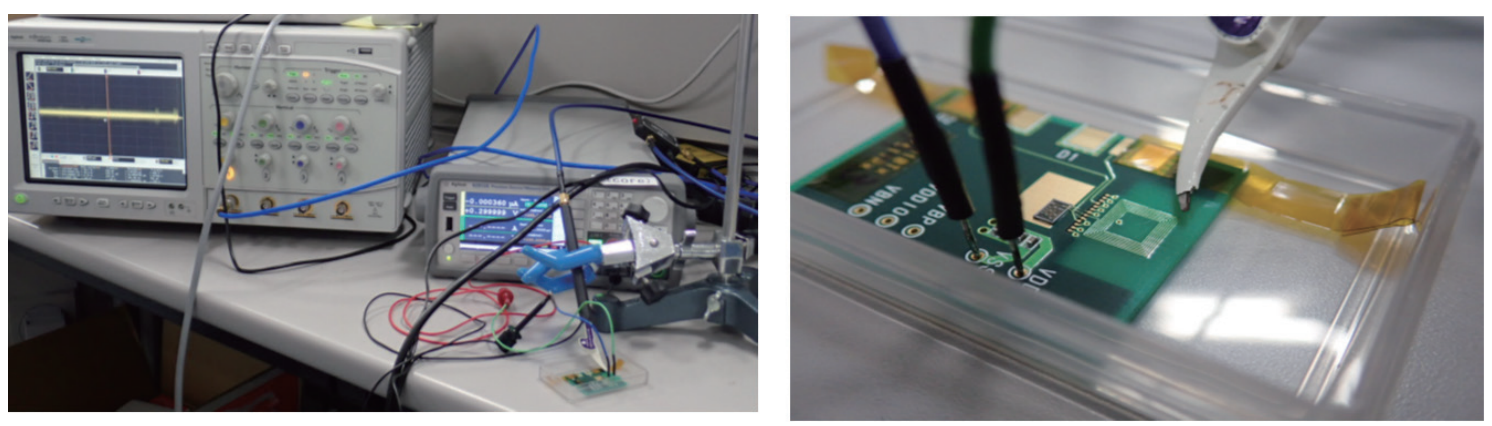

Fig. 12. (Color online) Measurement setup for evaluating proposed transmitter. ${ }^{(11)}$ 


\section{Measurement Results}

The results of the functional test and verification of the proposed technique are shown in this section. Figure 13 shows the measured output frequency of the proposed inductive-coupling transmitter as a function of the supply voltage. As seen in Fig. 13, the measurement results are in agreement with the simulation results shown in Fig. 9. In addition, the output frequency increases with the supply voltage. Considering the application of the proposed technique to the supply-sensing architecture, a monotonic increase in output frequency with an increase in supply voltage is desirable because supply voltage varies with bioconcentration and the output frequency can contain information on bioconcentration. Therefore, the proposed inductive-coupling transmitter can be used as a transmitter for low-power supply-sensing architecture.

Figure 14 shows the measured total consumption power of the proposed and conventional inductive-coupling transmitters as a function of the supply voltage. The blue circles and blue solid line indicate the results of the proposed inductive-coupling transmitter and its approximate curve, respectively. The red squares and red dotted line indicate the results of the conventional transmitter and its approximate curve, respectively. As shown in Fig. 14, the power consumption of the conventional transmitter increases with the supply voltage, whereas the proposed transmitter exhibits flat power characteristics for supply voltages ranging from 0.5 to $0.58 \mathrm{~V}$. In other words, the power consumption of the proposed transmitter is low at high input voltages. This is because the auxiliary driving transistor does not function when the supply voltage is greater than $0.5 \mathrm{~V}$. Although the power consumption of the conventional transmitter decreases at $0.59 \mathrm{~V}$, this can be regarded as a fluctuation in measurement, as in the conventional transmitter, and the architecture of the circuit dictates that there should be an increase in power consumption with the supply voltage. By introducing the auxiliary driving technique, the total power of the circuit is successfully reduced by $28 \%$ for a supply voltage of $0.6 \mathrm{~V}$. Moreover, if

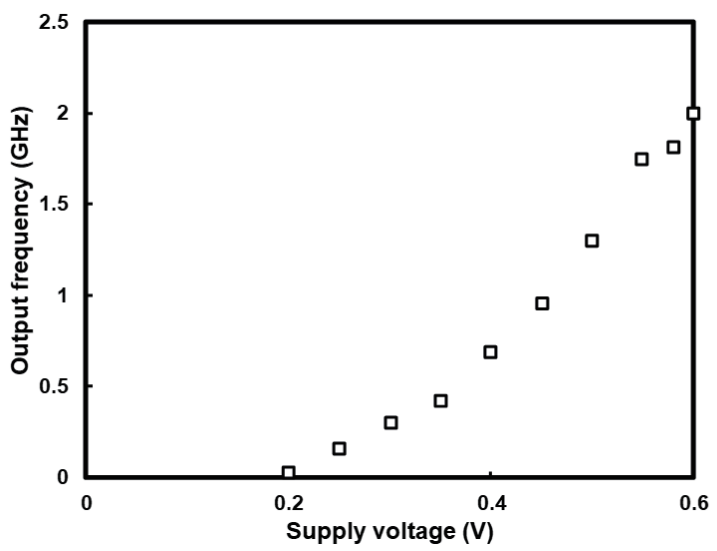

Fig. 13. Measured output frequency as a function of supply voltage of proposed transmitter. ${ }^{(11)}$

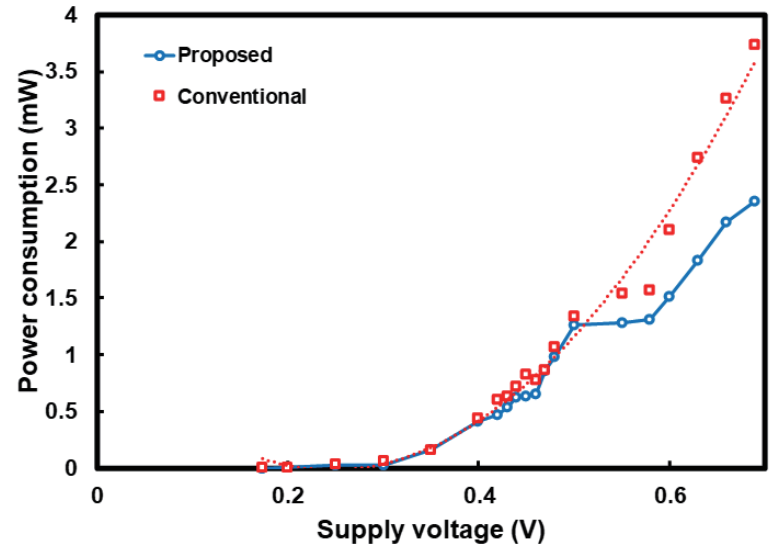

Fig. 14. (Color online) Measured power consumption of proposed (blue solid line) and conventional (red dotted line) transmitters as a function of supply voltage. ${ }^{(11)}$ 
the supply voltage is maintained between 0.5 and $0.6 \mathrm{~V}$, the rate of increase in the power of the proposed transmitter is $2.5 \mathrm{~mW} / \mathrm{V}$, i.e., $33 \%$ of $7.6 \mathrm{~mW} / \mathrm{V}$, which is the rate of increase in the power of the conventional inductive-coupling transmitter.

In summary, the results show that the proposed technique is efficient for power reduction. In the future, we plan to further reduce the power consumption by increasing the number of auxiliary drivers. In addition, design optimizations, such as sizing the auxiliary driver, will contribute to power reduction.

\section{Discussion}

In this section, the advantages and disadvantages of the proposed technique are discussed. Table 1 shows the measurement results of the transmitters with and without an auxiliary driver. As described previously, the main advantage of the proposed technique is power reduction. The average and increase rates of the consumption power from $V_{D D}=0.5$ to $0.6 \mathrm{~V}$ could be reduced by 19 and $67 \%$, respectively. However, the proposed technique requires additional control circuits, leading to additional power consumption and footprint. Thus, the power can be reduced only when the total power consumption of the control circuits is lower than the reduced power of the driver. The proposed inductive-coupling transmitter with an auxiliary driving transistor could suppress the increase in power consumption with supply voltage, as compared with the conventional inductive-coupling transmitter. ${ }^{(9)}$

Typically, the power consumed by the driver accounts for a significant portion of the total power consumption. Therefore, the advantages are guaranteed to outweigh the disadvantages under typical operational conditions. Although an increase in footprint is inevitable, the advantages afforded by the proposed technique outweigh the disadvantages of the overhead of the footprint of less than $10 \%$.

Moreover, the total power consumed by the control circuits can be reduced by technology scaling. Thus, the power consumed by the control circuits becomes increasingly less dominant with technology scaling. The additional footprint also becomes less dominant because the control circuits consist of logic gates. This means that the proposed technique is effective in a typical scenario and beneficial for biomedical IoT applications. ${ }^{(14-18)}$

Table 1

Measurement results of inductive-coupling transmitters

\begin{tabular}{lccc}
\hline & \multicolumn{2}{c}{ This work } & TCAS-I 2018 \\
\hline Architecture & $\begin{array}{c}\text { Without } \\
\text { auxiliary driver }\end{array}$ & $\begin{array}{c}\text { With } \\
\text { auxiliary driver }\end{array}$ & $\begin{array}{c}\text { Without } \\
\text { auxiliary driver }\end{array}$ \\
\hline Total area $\left(\mathrm{mm}^{2}\right)$ & 160 & 180 & 7200 \\
\hline $\begin{array}{l}\text { Power consumption } \\
@, V_{D D}=0.5 \mathrm{~V}(\mathrm{~mW})\end{array}$ & 1.34 & 1.26 & 1.07 \\
\hline $\begin{array}{l}\text { Power consumption } \\
@ \quad V_{D D}=0.6 \mathrm{~V}(\mathrm{~mW})\end{array}$ & 2.10 & 1.51 & 1.66 \\
\hline $\begin{array}{l}\text { Average power consumption } \\
\text { from } V_{D D}=0.5 \text { to } 0.6 \mathrm{~V}(\mathrm{~mW})\end{array}$ & 1.64 & 1.33 & 1.37 \\
\hline $\begin{array}{l}\text { Power consumption increase rate } \\
\text { from } V_{D D}=0.5 \text { to } 0.6 \mathrm{~V}(\mathrm{~mW} / \mathrm{V})\end{array}$ & 7.6 & 2.5 & 5.9 \\
\hline
\end{tabular}




\section{Conclusion}

An auxiliary driving technique has been proposed to realize a constant-power inductive-coupling transmitter for low-power supply-sensing biosensing platforms. The test chip fabricated using $65 \mathrm{~nm}$ SOTB CMOS technology successfully demonstrated the constant-power transmission capability of the inductive-coupling transmitter fabricated by the proposed technique, while the power consumed by the conventional inductive-coupling transmitter increases with the supply voltage. This technique is effective in a typical technology scaling scenario and beneficial for biomedical applications.

\section{Acknowledgments}

This research was financially supported by JST, PRESTO, through Grants-in-Aid for Scientific Research (S) (Nos. 20226009 and 25220906), by a Grant-in-Aid for Young Scientists (A) (No. 16H06088) from the Ministry of Education, Culture, Sports, Science and Technology of Japan, by the Strategic Information and Communications R\&D Promotion Programme (Nos. 121806006 and 152106004) of the Ministry of Internal Affairs and Communications, Japan, by TOYOTA RIKEN, and by The Nitto Foundation. The fabrication of CMOS chips was supported by Taiwan Semiconductor Manufacturing Co., Ltd. (TSMC, Taiwan), the VLSI Design and Education Center (VDEC), and the University of Tokyo in collaboration with Synopsys, Inc. and Cadence Design Systems, Inc.

\section{References}

1 H. Hafezi, T. L. Robertson, G. D. Moon, K. Au-Yeung, M. J. Zdeblick, and G. M. Savage: IEEE Trans. Biomed. Eng. 62 (2015) 99. https://doi.org/10.1109/TBME.2014.2341272

2 D. Seo, R. M. Neely, K. Shen, U. Singhal, E. Alon, J. M. Rabaey, J. M. Carmena, and M. M. Maharbiz: Neuron 91 (2016) 529. https://doi.org/10.1016/j.neuron.2016.06.034

3 D. Seo, J. M. Carmena, J. M. Rabaey, M. M. Maharbiz, and E. Alon: J. Neurosci. Methods 244 (2015) 114. https://doi.org/10.1016/j.jneumeth.2014.07.025

4 X. Chen, X. Zhang, L. Zhang, X. Li, N. Qi, H. Jiang, and Z. Wang: IEEE Trans. Biomed. Circuits Syst. 3 (2009) 11. https://doi.org/10.1109/TBCAS.2008.2006493

5 S. B. Lee, H. Lee, M. Kiani, U. Jow, and M. Ghovanloo: IEEE Trans. Biomed. Circuits Syst. 4 (2010) 360. https://doi.org/10.1109/TBCAS.2010.2078814

6 A. Roy, A. Klinefelter, F. B. Yahya, X. Chen, L. P. Gonzalez-Guerrero, C. J. Lukas, D. A. Kamakshi, J. Boley, K. Craig, M. Faisal, S. Oh, N. E. Roberts, Y. Shakhsheer, A. Shrivastava, D. P. Vasudevan, D. D. Wentzloff, and B. H. Calhoun: IEEE Trans. Biomed. Circuits Syst. 9 (2015) 862. https://doi.org/10.1109/TBCAS.2015.2498643

7 S. Arata, K. Hayashi, Y. Nishio, A. Kobayashi, K. Nakazato, and K. Niitsu: Jpn. J. Appl. Phys. 57 (2018) 04FM04. https://doi.org/10.7567/JJAP.57.04FM04

8 R. Cabrera, I. Weaver, A. Banerjee, R. Sarpeshkar, and T. Thorsen: ECS Trans. 72 (2016) 31. https://doi. org $/ 10.1149 / 07203.0031$ ecst

9 K. Niitsu, A. Kobayashi, Y. Nishio, K. Hayashi, K. Ikeda, T. Ando, Y. Ogawa, H. Kai, M. Nishizawa, and K. Nakazato: IEEE Trans. Circuits Syst. I 65 (2018) 2784. https://doi.org/10.1109/TCSI.2018.2791516

10 K. Hayashi, S. Arata, S. Murakami, Y. Nishio, A. Kobayashi, and K. Niitsu: IEEE Trans. Circuits Syst. II 65 (2018) 1360. https://doi.org/10.1109/TCSII.2018.2860636

11 Y. Nishio, A. Kobayashi, and K. Niitsu: Proc. IEEE Asia Pacific Conf. Circuits and Systems (2018) 65. https:// doi.org/10.1109/APCCAS.2018.8605645

12 K. Niitsu, Y. Shimazaki, Y. Sugimori, Y. Kohama, K. Kasuga, I. Nonomura, M. Saen, S. Komatsu, K. Osada, N. Irie, T. Hattori, A. Hasegawa, and T. Kuroda: Proc. IEEE Int. Solid-State Circuits Conf. (2009) 480. https:// doi.org/10.1109/ISSCC.2009.4977517 
13 N. Miura, D. Mizoguchi, M. Inoue, K. Niitsu, Y. Nakagawa, M. Tago, M. Fukaishi, T. Sakurai, and T. Kuroda: IEEE J. Solid-State Circuits, 42 (2007) 111. https://doi.org/10.1109/JSSC.2006.886554

14 K. Niitsu, T. Nakanishi, S. Murakami, M. Matsunaga, A. Kobayashi, N. M. Karim, J. Ito, N. Ozawa, T. Hase, H. Tanaka, M. Sato, H. Kondo, K. Ishikawa, H. Odaka, Y. Hasegawa, M. Hori, and K. Nakazato: IEEE Trans. Biomed. Circuits Syst. 13 (2019) 470. https://doi.org/10.1109/TBCAS.2018.2882472

15 M. Matsunaga, T. Nakanishi, A. Kobayashi, K. Nakazato, and K. Niitsu: Analog Integ. Circuits Signal Process. 98 (2019) 453. https://doi.org/10.1007/s10470-018-1267-5

16 M. Matsunaga, A. Kobayashi, K. Nakazato, and K. Niitsu: Jpn. J. Appl. Phys. 57 (2018) 03EC02. https://doi. org/10.7567/JJAP.57.03EC02

17 Y. Nishio, A. Kobayashi, and K. Niitsu: IEICE Trans. Electron. E102-C (2019) 269. https://doi.org/10.1587/ transele.2018CDP0005

18 A. Kobayashi, K. Ikeda, Y. Ogawa, H. Kai, M. Nishizawa, K. Nakazato, and K. Niitsu: IEEE Trans. Biomed. Circuits Syst. 11 (2017) 1313. https://doi.org/10.1109/TBCAS.2017.2735447

\section{About the Authors}
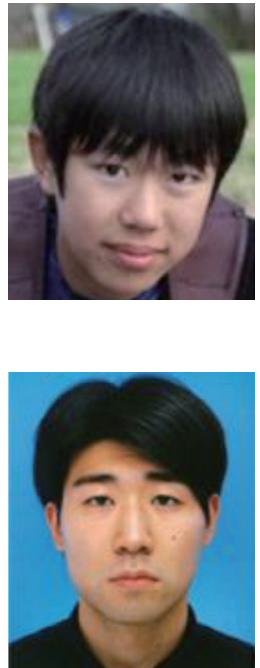

Yuya Nishio was born in Japan in 1996. He received his B.S. degree in electrical engineering and computer science in 2019 from Nagoya University, Japan, where he is currently working toward his M.S. degree. His research interests include mixed-signal CMOS integrated circuits, emerging nanotechnologies, and emerging biomedical applications.

(nishio.yuya@b.mbox.nagoya-u.ac.jp)

Atsuki Kobayashi was born in Yamanashi, Japan in 1993. He received his B.S. and M.S. degrees in electrical engineering and computer science in 2016 and 2018, respectively, from Nagoya University, Nagoya, Japan, where he is currently working toward his Ph.D. degree. His research activity is focused on mixed-signal CMOS integrated circuits for biomedical applications. He was the recipient of the IEEE Biomedical Circuits and Systems Conference (BioCAS) 2016 Best Paper Award. (kobayashi.atsuki@d.mbox.nagoya-u.ac.jp)

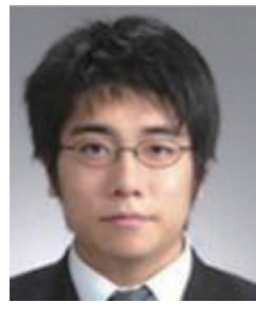

Kiichi Niitsu was born in Japan in 1983. He received his B.S. (summa cum laude), M.S., and Ph.D. degrees in electrical engineering from Keio University, Yokohama, Japan, in 2006, 2008, and 2010, respectively. From 2008 to 2010, he was a research fellow of the Japan Society for the Promotion of Science, a research assistant of the Global Center of Excellence Program, Keio University, and a collaboration researcher at the Keio Advanced Research Center. In 2010, he became an assistant professor at Gunma University, Kiryu, Japan. In 2012, he became a lecturer at Nagoya University, Nagoya, Japan. Since 2015, he has been a researcher at Precursory Research for Embryonic Science and Technology, Japan Science and Technology Agency. His current research interests lie in low-power and high-speed technologies of analog and digital VLSI circuits for biomedical applications. He was a recipient of the 2006 KEIO KOUGAKUKAI Award, the 2007 INOSE Science Promotion Award, the 2008 IEEE SSCS Japan Chapter Young Researcher 
Award, the 2009 IEEE SSCS Japan Chapter Academic Research Award from IEEE Solid-State Circuits Society Japan Chapter, the 2008 FUJIWARA Award from the FUJIWARA foundation, the 2011 YASUJIRO NIWA Outstanding Paper Award, the 2011 FUNAI Research Promotion Award, the 2011 Ando Incentive Prize for the Study of Electronics, the 2011 Ericsson Young Scientist Award, the 2012 ASP-DAC University LSI Design Contest Design Award, the NF Foundation Research and Development Encouragement Award, the AKASAKI Award from Nagoya University, the IEEE Nagoya Section Young Researcher Award, the IEEE Biomedical Circuits and Systems Conference 2016 (BioCAS 2016) Best Paper Award, the 2017 Commendation for Science and Technology by the Minister of Education, Culture, Sports, Science and Technology, and the Young Scientists Prize, the 2018 SUEMATSU-Yasuharu Award from IEICE. He has authored or co-authored 51 referred original journal papers, 111 international conference papers, and three book chapters, including two TBioCAS, one TCAS-I, five JSSC, five TVLSI, two ISSCC, four Symp. VLSI Circuits, nine BioCAS, one ISCAS, and four A-SSCC. He has served as a technical committee member of the IEEE Biomedical Circuits and Systems (BioCAS TC), a review committee member of ISCAS 2017/2018, a technical program committee member of ICECS 2018, a review committee member of APCCAS 2014, an editorial committee member of IEICE Transactions on Electronics, Special Section on Analog Circuits and Related SoC Integration Technologies, and an editorial committee member of IEICE ESS Fundamental Review. He is a member of the Institute of Electronics, Information and Communication Engineers of Japan and the Japan Society of Applied Physics. (niitsu@nuee.nagoya-u.ac.jp) 